\title{
Observer-based actuator fault detection for UAV XUE Ting*
}

(School of Instrumentation Science and Optoelectronics Engineering, Beihang University, China) email: xuet_buaa@126.com

Keywords: UAV, Actuator, Fault detection, Observer, $H_{i} / H_{\infty}$

Abstract. This paper focuses on the actuator fault detection for UAV. Firstly, the longitudinal dynamics model of UAV with actuator faults and atmosphere turbulence is given. Based on this, an observer-based fault detection filter is applied for residual generation, and a $H_{i} / H_{\infty}$ performance index is introduced, which describes the sensitivity to fault as well as the robustness against unknown disturbance, then the design of residual generator is formulated as an $H_{i} / H_{\infty}$ optimization problem. And then, the 2-norm based residual evaluation function and the corresponding threshold are used for residual evaluation. Finally, the simulations with certain type of UAV are presented to verify the effectiveness of the proposed method.

\section{Introduction}

Nowadays, unmanned aerial vehicle (UAV) is widely used in the fields of research and practical, due to its particular features. Meanwhile, the security and reliability of UAV flight control system (FCS) attracts much attention throughout the world [1]. The actuators are the key part of FCS, and play an important role to guarantee the security and reliability of UAV. However, in long endurance flight mission, actuators inevitably surfing from some faults such as stuck or partial damage etc., the serious situation would leads to the crash accident. One promising approach to improving the safety and reliability of UAV is employing the fault detection method [2].

Many researches have been done at home and abroad for the problem of actuator fault detection [1]-[3]. Among these achievements, observer-based fault detection (FD) proves to be an effective method. Its basic idea is to generate a residual by designing a fault detection filter (FDF), and then the suitable residual evaluation function and threshold are selected to evaluate the residual. For the aircraft would face all kinds of unknown disturbances, like atmosphere turbulence, random noise and so on. So the robust fault detection for UAV has become an important aspect of research. One salutation is to design a FDF, which is decoupled from disturbances, e.g. [3]. While it's difficult to satisfy the conditions of decouple in real systems. Recently, another more commonly used method is $H_{i} / H_{\infty}$ optimization technology. It employs $H_{i}$ norm and $H_{\infty}$ norm to describe the sensitivity to fault and the robustness against the unknown disturbances, respectively, and based on this, the FDF design is formulated to the optimal $H_{i} / H_{\infty}$ performance index problem. This method can achieve a good tradeoff between the sensitivity to fault and robustness against disturbances, and a unified solution has been given for linear system in [4], which is easy to implement in practical.

Considering the practical needs of actuator fault detection for UAV, this paper applies the optimal $H_{i} / H_{\infty}$ based method for FDF design. Firstly, the actuator faults are disturbed as additive faults, and the longitudinal dynamics model with actuator faults and disturbances is given. Then the FDF is designed based on optimal $H_{i} / H_{\infty}$ based method, and the residual evaluation function and threshold based on 2-norm are determined for residual evaluation. Finally, the simulations with certain type of UAV are illustrated to verify the effectiveness of the proposed approach.

\section{Longitudinal Dynamics of UAV with actuator fault}

Here a fixed-wing UAV is considered. The UAV model is based around the six-degree-freedom nonlinear aircraft model. By using the small perturbation theory, the nonlinear 
model can be linearized. Considering the atmosphere turbulence disturbance, the linearized longitudinal dynamics equations of the UAV is [5]

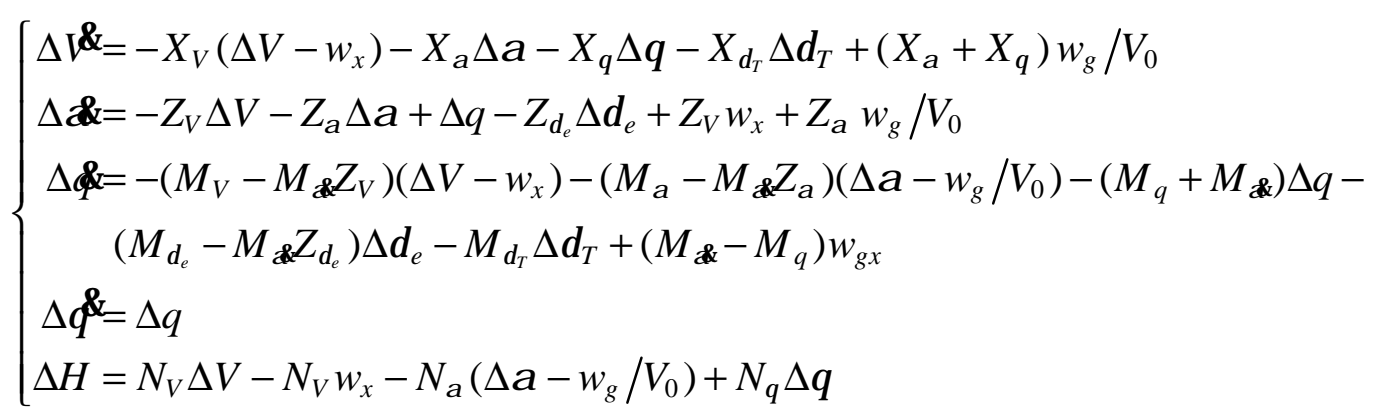

Where $V_{0}$ is the velocity of the aircraft; $\Delta V, \Delta \alpha, \Delta q, \Delta \theta$ and $\Delta H$ are the changes of the aircraft velocity, attack angle, pitching angle, pitching angle rate and height, respectively; $\Delta \delta_{e}$ and $\Delta \delta_{T}$ are the changes of elevator and thrust, respectively; $w_{x}$ and $w_{g}$ are the turbulence velocity; $w_{g x}$ is the gradient of $w_{g}$ along the $X$ axis; $X_{V}, X_{\alpha}, X_{\theta}, X_{\delta_{T}}, Z_{V}, Z_{\alpha}, Z_{\theta}, Z_{\delta_{e}}, M_{V}, M_{\alpha}, M_{\alpha \delta_{e}} M_{q}, M_{\delta_{T}}, M_{\delta_{e}}, N_{V}, N_{\alpha}, N_{\theta}$ are the constant parameters, which are determined by the aircraft configuration parameters and the aerodynamic derivatives in steady state flight.

Actuators are the important parts to guarantee the the security and reliability of aircraft, but they inevitably face unexpected incidents, such as actuator stuck or the rudder damage etc.. The typical actuator faults include stuck, damage and basis fault. Without loss of generality, these faults can be modeled as additive faults. For the FCS is closed-loop system, the introduction of a feedback control makes the impact caused by the early failure or the small amplitude faults masked by the control function. For this reason, it becomes more difficult to detect the actuator faults for UAV [6].

Here the system state variables are selected as $x=\left[\begin{array}{llll}\Delta V & \Delta \alpha & \Delta \theta & \Delta H\end{array}\right]^{T}$, the input variables are $u=\left[\begin{array}{ll}\Delta \delta_{e} & \Delta \delta_{P}\end{array}\right]^{T}$, the output variables are $y=\left[\begin{array}{lll}\Delta V & \Delta \bar{\alpha} \Delta \bar{q} \Delta \bar{\theta} \Delta H\end{array}\right]^{T}$, the unknown disturbances are collectively described as $d=\left[\begin{array}{ll}d_{w}^{T} & v^{T}\end{array}\right]^{T}$, where $v$ is the measurements noise , $d_{w}=\left[\begin{array}{lll}w_{x} & w_{g} & w_{g x}\end{array}\right]^{T}$ is the atmosphere turbulence disturbance. Then the longitudinal model of UAV in the state space form is

$$
\left\{\begin{array}{l}
f(t)=A x(t)+B u(t)+E_{d} d(t)+B f(t) \\
y(t)=C x(t)+F_{d} d(t)
\end{array}\right.
$$

Where $x \in R^{k_{x}}, u \in R^{k_{u}}, y \in R^{k_{y}}, A 、 B 、 C$ are the known system coefficient matrixes, $E_{d}=\left[\begin{array}{ll}B_{d} & 0\end{array}\right]$, $F_{d}=\left[\begin{array}{ll}0 & I_{k_{y}}\end{array}\right], I_{k_{y}}$ is a unit matrix, $\boldsymbol{O}$ is a zeros matrix, $B_{d}$ is the turbulence coefficient matrix.

\section{Design of actuator fault detection system for UAV}

Considering the longitudinal dynamic system mentioned in Eq. (1), in general, it is always reasonable to assume that the unknown disturbance $d$ is $L_{2}$ norm bounded, $(C, A)$ is observable, and $\left[\begin{array}{cc}A-j \omega I & E_{d} \\ C & F_{d}\end{array}\right]$ has full row rank for all $\omega \in[0, \infty)$. Then a FDF based on observer is designed as follows to generate residual:

$$
\left\{\begin{array}{l}
\hat{f}(t)=A \hat{x}(t)+B u(t)+L(y(t)-\hat{y}(t)) \\
\hat{y}(t)=C \hat{x}(t) \\
r(t)=R(s)(y(t)-\hat{y}(t))
\end{array}\right.
$$


Where $r$ is the residual signal, $\hat{x}, \hat{y}$ are the estimates of $x, y$, respectively; $L$ and $R(s)$ are the observer gain matrix and the post-filter to be designed, respectively.

By defining the state estimation error $e(t)=x(t)-\hat{x}(t)$, it follows from Eq. (2) and Eq. (3) that

$$
\left\{\begin{array}{l}
\mathscr{E}(t)=(A-L C) e(t)+\left(E_{d}-L F_{d}\right) d(t)+B f(t) \\
r(t)=R(s)\left(C e(t)+F_{d} d(t)\right)
\end{array}\right.
$$

In frequency domain, the residual can be represented as

$$
r(s)=R(s)\left(G_{d m}(s) d(s)+G_{f m}(s) f(s)\right)
$$

Where $G_{d m}(s)=C(s I-A+L C)^{-1}\left(E_{d}-L F_{d}\right)+F_{d}, \quad G_{f m}(s)=C(s I-A+L C)^{-1} B \quad$ are the transfer functions from $d$ and $f$ to $r$.

For the real UAV system, it's almost impossible to decouple disturbances $d$ from the residual. A promising method is to employ the $H_{i} / H_{\infty}$ performance index, in which the sensitivity to fault is evaluated by the non-zero singular value $\sigma_{i}\left(R(j \omega) G_{f m}(j \omega)\right)$, and the robustness against unknown disturbances is evaluated by the $H_{\infty}$-norm $\left\|R(s) G_{d m}(s)\right\|_{\infty}$. Then the FDF design is converted to the following $H_{i} / H_{\infty}$ performance index optimization problem:

$$
\sup _{L, R} J_{i / \infty}=\sup _{L, R} \frac{\sigma_{i}\left(R(j \omega) G_{f m}(j \omega)\right)}{\left\|R(s) G_{d m}(s)\right\|_{\infty}}, \quad \omega \in[0, \infty)
$$

For this optimization problem, a unified solution has been given in [4], just as follows:

$$
L_{o p t}=\left(E_{f} F_{f}^{T}+Y C^{T}\right)\left(F_{d} F_{d}^{T}\right)^{-1}, R_{o p t}=\left(F_{d} F_{d}^{T}\right)^{-1 / 2}
$$

Where $Y \geq 0$ is a solution of algebraic Riccati equation

$$
A Y+Y A^{T}+E_{d} E_{d}^{T}-\left(E_{d} F_{d}^{T}+Y C^{T}\right)\left(F_{d} F_{d}^{T}\right)^{-1}\left(F_{d} E_{d}^{T}+C Y\right)=0
$$

By solving the Riccati equation, the optimal solutions $\left(L_{\text {opt }}, R_{\text {opt }}\right)$ can be obtained. Through bringing $\left(L_{\text {opt }}, R_{\text {opt }}\right)$ into Eq. (3), the design of residual generator is accomplished.

In the stage of residual evaluation, the residual evaluation function $J(r)$ based on 2-norm of residual is used. For the evaluation just within finite time is realizable in practical applications, the residual evaluation function $J(r)$ is defined as

$$
J(r)=\|r\|_{2, T}=\sqrt{\int_{t_{1}}^{t_{2}} r^{T}(t) r(t) d t}, T=t_{2}-t_{1}
$$

In fault-free cases, the corresponding threshold $J_{t h}$ is confirmed as follows:

$$
J_{t h}=\sup _{d \in L_{2}, f=0}\|r\|_{2}=\sup \left\|R(j \omega) G_{d m}(j \omega) d(t)\right\|_{2}
$$

By defining $\gamma=\left\|R(j \omega) G_{d m}(j \omega)\right\|_{\infty},\|d\|_{2} \leq \delta$, according to the unified solutions, $\gamma=1$. there exists the following norm inequality 


$$
\sup \left\|R(j \omega) G_{d m}(j \omega) d(t)\right\|_{2} \leq\left\|R(j \omega) G_{d m}(j \omega)\right\|_{\infty} \sup \|d\|_{2}=\gamma \delta
$$

According to above analysis, the threshold is confirmed to be $J_{t h}=\delta$. The decision logic of fault detection is shown as follows:

$$
\begin{cases}J(r) \leq J_{t h}, & \text { fault-free; } \\ J(r)>J_{t h}, & \text { fault }\end{cases}
$$

\section{Simulation Results}

In order to verify the effectiveness of the presented method, simulations are carried on in MATLAB environment with certain type of UAV. The system coefficient matrices shown in (3) respectively are

$$
A=\left[\begin{array}{ccccc}
-0.0671 & 9.105 & 0 & -9.8 & 0 \\
-0.0314 & -2.60 & 0.979 & 0 & 0 \\
0.0132 & -5.37 & -0.965 & 0 & 0 \\
0 & 0 & 1 & 0 & 0 \\
0 & -24 & 0 & 24 & 0
\end{array}\right] B=\left[\begin{array}{ccc}
0 & 0.09461 \\
-0.0020 & 0 \\
-0.1475 & 0 \\
0 & 0 \\
0 & 0
\end{array}\right] C=I_{5} B_{d}=\left[\begin{array}{cc}
-0.0671 & 0.029 \\
-0.0314 & 0.108 \\
0.0132 & 0.224 \\
0 & 0 \\
0 & -1
\end{array}\right]
$$

Consider the measurements noise is a white noise vector, and every element is uniformly distributed between $[-0.1,0.1]$. Ignoring the gradient disturbance $w_{g x}$, and the Dryden model is used to describe the turbulence velocity $w_{x}$ and $w_{g}$ for simulation purposes, i.e.

$$
w_{x}=w_{g}=\sqrt{\frac{3 V_{0} \sigma_{w}^{2}}{\pi L_{w}}} \frac{V_{0} /\left(\sqrt{3} L_{w}\right)+s}{\left[V_{0} / L_{w}+s\right]^{2}} n
$$

Where $n$ is a zero-mean Gaussian white noise, the turbulence scale is $L_{w}=480 m$, and the turbulence intension is $\sigma_{w}=6 \mathrm{~m} / \mathrm{s}$. Moreover, the disturbance satisfies $\|d\|_{2} \leq \delta=1.021$.

The simulations are realized in 100 seconds with 0.01 seconds of sampling time. The evaluation window is set as $T=1 s$. To test the effectiveness of proposed method, following cases are considered.

Case (1): the elevator actuator gets stuck at 20s, and the amplitude of the fault is $0.1^{\circ}$, the detection result is shown on Fig.1.

Case (2): for the reasons of elevator surface damage or icing, the execution efficiency of actuator decline $10 \%$ during 20 40s and decline 30\% during 60 80s, the detection result is shown on Fig.2.

Case (3): the elevator actuator occurs $0.1^{\circ}$ constant basis fault from $20 \mathrm{~s}$ to $60 \mathrm{~s}$, the detection result is shown on Fig.3.

Case (4): the elevator actuator occurs time-varying basis fault during 20 60s, whose amplitude is $0.1^{\circ}$ and the frequency is set $0.2 \mathrm{~Hz}$, the detection result is shown on Fig. 4 . 


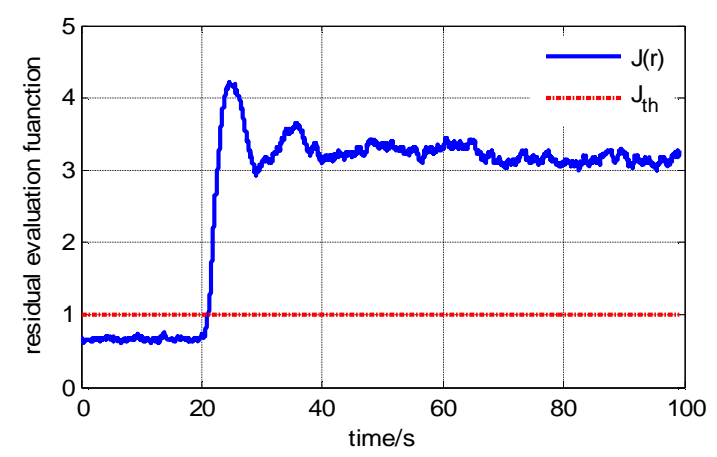

Fig.1 Elevator $0.1^{\circ}$ stuck fault detection

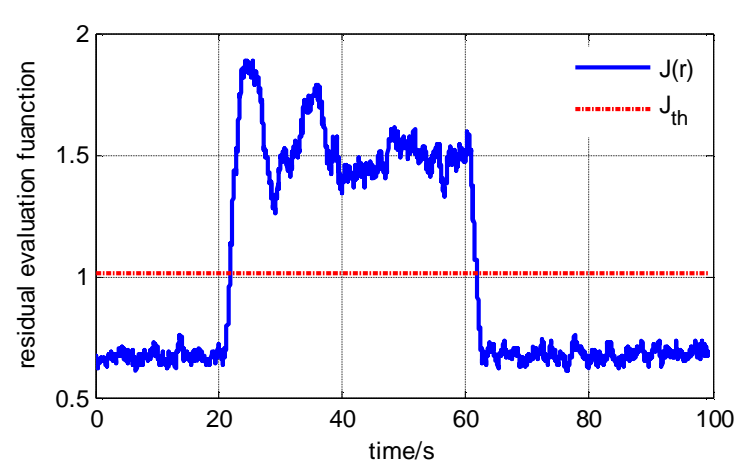

Fig.3 Elevator $0.1^{\circ}$ basis fault detection

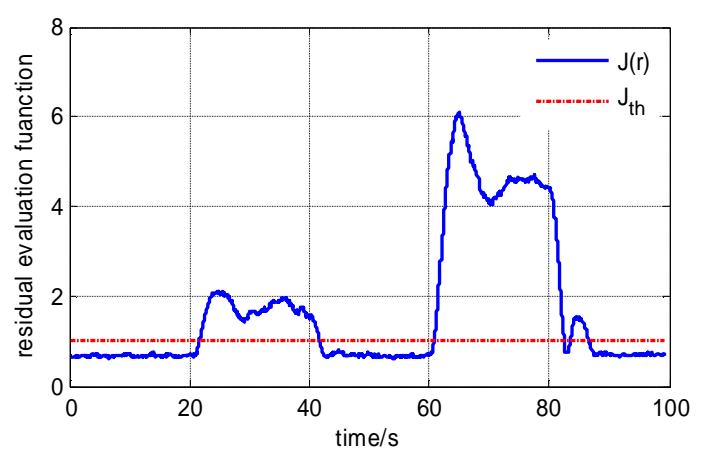

Fig.2 Elevator $10 \%$ and $30 \%$ damage fault detection

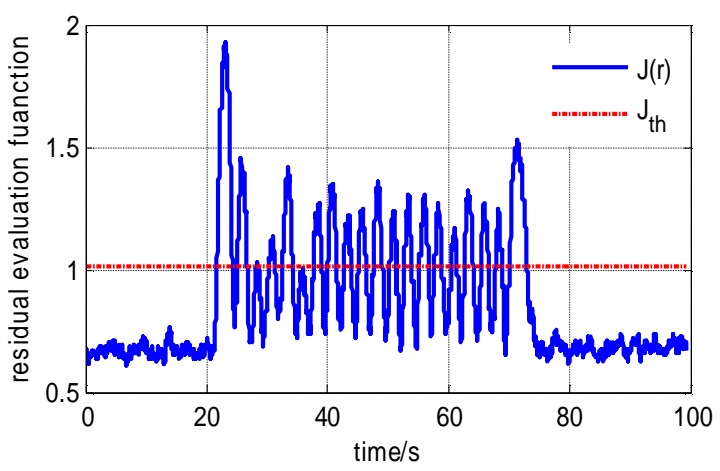

Fig.4 Elevator 0.2Hz time-varying basis fault detection

From the above simulation results shown on the figures from one to four, it is seen that the proposed method can obviously detected different kinds of actuator faults, such as stuck fault, partial damage fault, constant basis fault and time-varying basis fault. Meanwhile, the detection time-delay is small, which means a good real-time of fault detection system. In addition, the design of fault detection system is as simple as to be employed in practical applications.

\section{Summary}

In this paper, a simultaneous fault detection method has been proposed to detect actuator faults for UAV. The longitudinal dynamics model of UAV was given firstly, and the actuator faults can be considered as an additive faults. Based on this, the fault model was built. For the purpose of fault detection, a fault detection filter was designed with the optimal $H_{i} / H_{\infty}$ performance index. And then, a 2-norm based residual evaluation function and threshold were introduced for residual evaluation to realize the actuator fault detection. Finally, the simulation results show that, this method can simultaneously detect the actuator faults effectively and fleetly. Moreover, the design and implement of the fault detection system is simple in practical applications.

\section{References}

[1] B. Hu, P. Seiler. Pivotal decomposition for reliability analysis of fault tolerant control systems on unmanned aerial vehicles[J]. Reliability Engineering \& System Safety, 140(1): 130-14. (2015).

[2] X. Qi, J. T. Qi, D. Theilliol, et al. A review on fault diagnosis and fault tolerant control methods for single-rotor aerial vehicles[J]. Intelligent \& Robot System, 73(1), pp. 535-555. (2014).

[3] J. Xu, K. M. Peng. FDI UIOs with multiple considerations for flight systems: an LMI approach[C] // The 7th IEEE Conference on Industrial Electronics and Applications, IEEE, pp. 588-593. ( 2012).

[4] S. X. Ding. Model-based fault diagnosis techniques[M]. Berlin: Springer. ( 2013). 
[5] L. Y. Wen, G. Tao, H. Yang. Aircraft flight system models under turbulence conditions[C], //The 33rd Chinese Control Conference, pp. 8795-8800. (2014).

[6] D. H. Zhou, Y. Liu, X. He. Review on fault diagnosis techniques for closed-loop systems[J]. Acta Auto. Sinica, 39(11), pp. 1933-1943. (2013).

[7] G. H. Yang, H. Wang. Fault detection for a class of uncertain state-Feedback control system[J]. IEEE Trans. on Contr. Syst. Techno., 18(1), pp. 201-212. (2010). 